\title{
Laser-Particle Collider for Multi-GeV Photon Production
}

\author{
J. Magnusson, ${ }^{1, *}$ A. Gonoskov, ${ }^{2,3,4}$ M. Marklund, ${ }^{2}$ T. Zh. Esirkepov, ${ }^{5}$ J. K. Koga,${ }^{5}$ K. Kondo, ${ }^{5}$ M. Kando, \\ S. V. Bulanov, ${ }^{5,6,7}$ G. Korn, ${ }^{6}$ and S. S. Bulanov ${ }^{8}$ \\ ${ }^{1}$ Department of Physics, Chalmers University of Technology, SE-41296 Gothenburg, Sweden \\ ${ }^{2}$ Department of Physics, University of Gothenburg, SE-41296 Gothenburg, Sweden \\ ${ }^{3}$ Institute of Applied Physics, Russian Academy of Sciences, Nizhny Novgorod 603950, Russia \\ ${ }^{4}$ Lobachevsky State University of Nizhni Novgorod, Nizhny Novgorod, 603950, Russia \\ ${ }^{5}$ Kansai Photon Science Institute, National Institutes for Quantum and Radiological Science and Technology (QST), \\ 8-1-7 Umemidai, Kizugawa, Kyoto 619-0215, Japan \\ ${ }^{6}$ Institute of Physics ASCR, v.v.i. (FZU), ELI-Beamlines Project, 18221 Prague, Czech Republic \\ ${ }^{7}$ Prokhorov General Physics Institute RAS, Vavilov street 38, Moscow 119991, Russia \\ ${ }^{8}$ Lawrence Berkeley National Laboratory, Berkeley, California 94720, USA
}

(Received 30 November 2018; published 27 June 2019)

\begin{abstract}
As an alternative to Compton backscattering and bremsstrahlung, the process of colliding high-energy electron beams with strong laser fields can more efficiently provide both a cleaner and brighter source of photons in the multi-GeV range for fundamental studies in nuclear and quark-gluon physics. In order to favor the emission of high-energy quanta and minimize their decay into electron-positron pairs, the fields must not only be sufficiently strong, but also well localized. We here examine these aspects and develop the concept of a laser-particle collider tailored for high-energy photon generation. We show that the use of multiple colliding laser pulses with $0.4 \mathrm{PW}$ of total power is capable of converting more than $18 \%$ of multi$\mathrm{GeV}$ electrons passing through the high-field region into photons, each of which carries more than half of the electron initial energy.
\end{abstract}

DOI: 10.1103/PhysRevLett.122.254801

The building and planning of several multi-PW laser facilities [1-5] and the accessibility of PW-class systems [6] have recently stimulated a strong interest in theoretical analysis of processes caused by the radiation reaction and by the phenomena of strong-field quantum electrodynamics (QED). The clarification of various theoretical aspects [714] as well as the development of analytical [15] and numerical [16-22] approaches has been instrumental in revealing various peculiar effects such as stochasticity [2325], straggling [17,26], quantum quenching [27], trapping in traveling $[28,29]$ and standing electromagnetic (EM) waves $[25,30-33]$ and the alteration of ponderomotive effects $[25,34]$. These findings encouraged several promising proposals of both current $[35,36]$ and future experiments. This includes the creation of positron [37] and photon [3844] sources as well as probing fundamental aspects of QED and astrophysics by reaching extreme conditions $[45,46]$.

Apart from concepts of laser-based positron, gamma, and $\mathrm{x}$-ray sources [47-50], which may become favored through advances in laser wakefield acceleration (LWFA) (see

Published by the American Physical Society under the terms of the Creative Commons Attribution 4.0 International license. Further distribution of this work must maintain attribution to the author(s) and the published article's title, journal citation, and DOI.
Ref. [51] and references therein), it is reasonable to also consider the use of optimally focused laser fields as targets for electron beams available with conventional accelerators. As it is today possible to create laser fields of sufficient strength for the emission of photons with energies comparable to that of the electrons, this process can provide an interesting alternative to Compton backscattering (CBS) and bremsstrahlung, presently used in producing $\mathrm{GeV}$ photons for probing nuclear and quark-gluon physics [52].

In this Letter we examine and develop the concept of such a laser-particle collider, applicable with both LWFA and conventional accelerators. We show that the use of a dipole focusing [53-55] of multiple colliding laser pulses (MCLP) [56] makes sub-PW laser systems capable of converting more than $18 \%$ of multi-GeV electrons passing through the high-field region into photons with more than half of the electron initial energy. Assuming energies achievable at modern electron accelerators, we here target the production of photons in the energy range of a few to several tens of $\mathrm{GeV}$, which is different from previous proposals [40-44,47-50]. In contrast to CBS sources, the considered setup can produce dense $\left(\sim 10^{18} \mathrm{~cm}^{-3}\right)$ beams of multi-GeV photons, while also providing a clean environment free of neutrons and other heavy particles that are naturally produced with bremsstrahlung and are not easily removable by an external electromagnetic field. In addition, the concept provides the possibility for 
controlling the polarization of photons, which is not possible with bremsstrahlung in this energy range. This can be advantageous for experiments such as the exploration of the Delbrück scattering [57]. Note that the MCLP setup considered in our study is also motivated by other proposals including tests of nonperturbative QED [56,58], triggering and confining QED cascades [31,39,59-61] as well as achieving extreme electron-positron plasma states via stratification [45] and pinching [46].

Motivating estimates.-An electron interacting with a strong laser field emits high-energy photons through the process of nonlinear Compton scattering. The probability of converting an electron with energy $\varepsilon_{0}=\gamma m c^{2}$ into a photon carrying off a significant part of the electron's energy becomes large when the quantum nonlinearity parameter $\chi=\left|F_{\mu \nu} p^{\nu}\right| / E_{S}[62]$ reaches values of the order of unity (where $F$ is the electromagnetic field tensor, $p$ is the particle four-momentum, and $E_{S}$ is the Sauter-Schwinger field). Using a standing wave structure, e.g., through MCLP, provides a geometry that maximizes $\chi$ such that it can be estimated as $\gamma a_{0} / a_{S}$. We use relativistic units for both the laser field $a_{0}=e E / m \omega c$ and the Sauter-Schwinger field $a_{S}=e E_{S} / m \omega c \approx 4.1 \times 10^{5} \lambda / 1 \mu \mathrm{m}$. Here $\hbar$ is the Plank constant, $c$ is the speed of light, $\omega$ and $\lambda$ are the laser frequency and wavelength, respectively, $e$ and $m$ are the charge and mass of the electron, respectively. Efficient conversion therefore occurs for $a_{0} \gtrsim a_{S} / \gamma$. If the electron experiences weaker fields, such that $\chi \ll 1$, but over an extended period of time its energy can be depleted through the emission of low-energy quanta $\left(\sim \chi \gamma m c^{2}\right)$. On the other hand, for sufficiently strong fields, a high-energy photon can in turn decay into an electron-positron pair, through multiphoton Breit-Wheeler pair production, as it propagates through the laser field. Optimal conversion of electrons into high-energy photons therefore requires a good field localization and some optimal field strength, which we will now assess with simple estimates.

We start from using high- $\chi$ approximation for the rates of these QED processes. We can then estimate the scale lengths for both the photon generation $l_{\text {rad }} \approx 15 \lambda_{C} \gamma^{1 / 3}\left(a_{0} / a_{S}\right)^{-2 / 3}$ and its decay $l_{\text {pair }} \approx 3 l_{\text {rad, }}$, where $\lambda_{C}=2.43 \times 10^{-10} \mathrm{~cm}$ is the Compton wavelength and where we in the latter estimate assume that the photon energy is $\gamma m c^{2} / 2$. As conversion efficiency, we will use the ratio $N_{1 / 2} / N$, where $N$ is the total number of electrons passing through the field and $N_{1 / 2}$ is the total number of photons with an energy above $\gamma m c^{2} / 2$ and that escape the interaction region. In order to maximize the yield according to this measure, one needs the field to be (i) sufficiently strong $(\chi \gtrsim 1)$ for generating high-energy photons and (ii) localized to within $\sim l_{\text {rad }}$ in order to hamper the conversion of the generated photons into pairs by the same field. The requirement (i) implies that $a_{0} \gtrsim a_{S} / \gamma$ and thus $l_{\text {rad }} \lesssim 15 \lambda_{C} \gamma$. For electron energies less than approximately $10 \mathrm{GeV}$ the value of $l_{\text {rad }}$ is always smaller than the optical wavelength $1 \mu \mathrm{m}$, which can be related to the

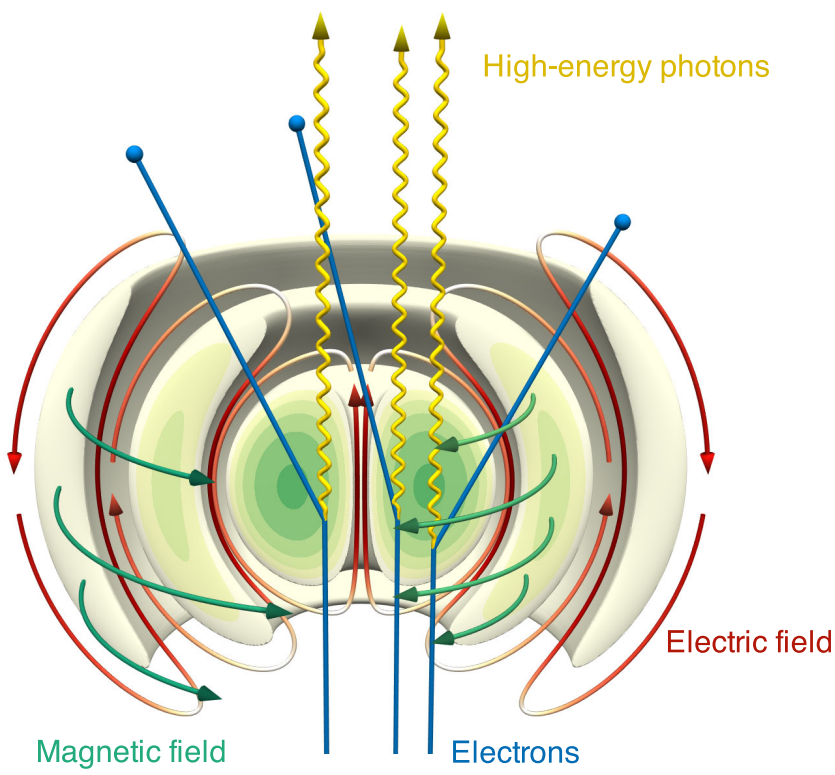

FIG. 1. Conceptual visualization of the setup, where highenergy electrons (blue) are injected along the axis of an intense dipole wave. In this field, the electrons will emit large amounts of high-energy photons (yellow). The polarization of the shown field is that of an electric dipole, with a poloidal electric field (red) and a toroidal magnetic field (green).

diffraction limit for the field localization. In this case, if we increase $a_{0}$ we first reach the regime of conversion into high-energy photons at around $a_{0} \sim a_{S} / \gamma$ (i.e., $\chi \sim 1$ ). As $a_{0}$ is increased further the number of escaped photons starts decreasing due to the decrease of $l_{\text {rad }}$. For energies higher than $10 \mathrm{GeV}$ we can make $l_{\text {rad }} \sim 1 \mu \mathrm{m}$ by setting the optimal field amplitude $a_{0} \approx 0.1 \gamma^{1 / 2}$, which yields $\chi>1$ and overall higher efficiency.

We see that the scale length of the field plays a crucial role in creating an efficient laser-particle collider for multi$\mathrm{GeV}$ photon production. A remarkable field localization is provided by the $4 \pi$-focusing geometry of a dipole wave [53] (see Fig. 1). For a certain phase, an electron moving at a distance of $\lambda / 3$ from and parallel to the dipole axis observes a field localized to within $0.3 \lambda$ (FWHM) and passes through the peak of the magnetic field at an amplitude of $\approx 500 \sqrt{P[\mathrm{PW}]}$, where $P$ is the total power of focused laser radiation. Using an averaged amplitude of $300 \sqrt{P[\mathrm{PW}]}$ over the size of this field we can estimate the optimal power from the conditions formulated above for low and high electron energies, and determine numerically the intermediate value from the data of Fig. 2:

$$
P^{\mathrm{opt}}\left(\varepsilon_{0}\right) \approx \begin{cases}0.5\left(\frac{\varepsilon_{0}}{1 \mathrm{GeV}}\right)^{-2} \mathrm{PW} & \text { if } \varepsilon_{0} \ll 10 \mathrm{GeV} \\ 0.4 \mathrm{PW} & \text { if } \varepsilon_{0} \sim 10 \mathrm{GeV} \\ \frac{\varepsilon_{0}}{160 \mathrm{GeV}} \mathrm{PW} & \text { if } \varepsilon_{0} \gg 10 \mathrm{GeV}\end{cases}
$$




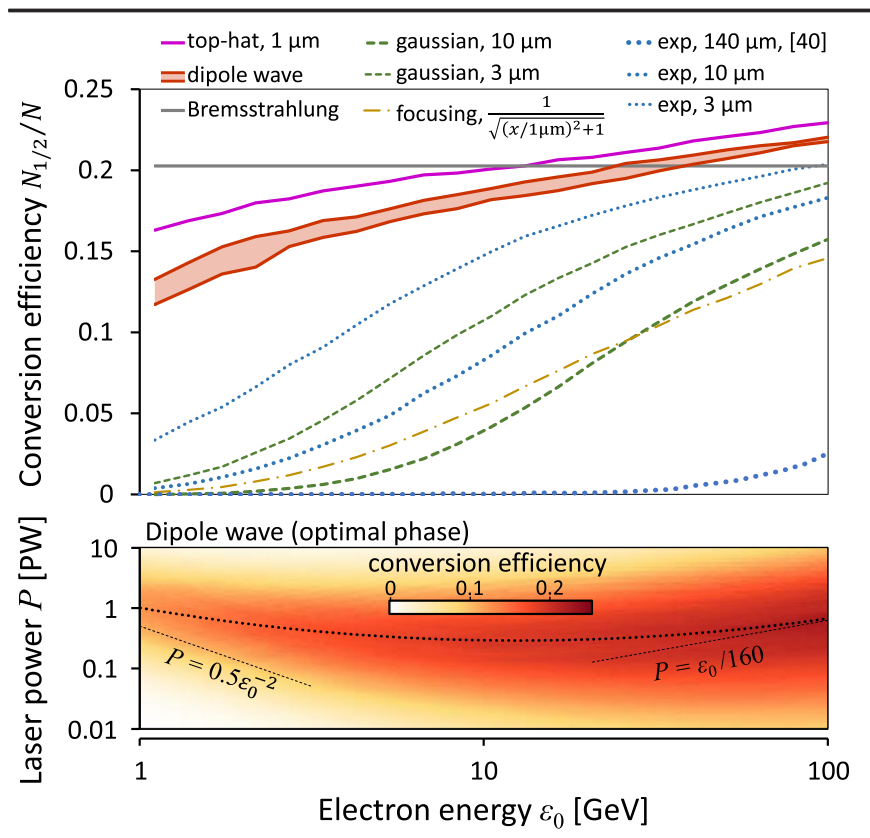

FIG. 2. The computed conversion efficiency for optimal amplitude as a function of initial electron energy for various shapes of the field amplitude $S(x)$ and scale lengths. The electrons and all generated particles are assumed to be ultrarelativistic and experience fields given by $a_{0} / a_{S}=S(c t)$. The case of $S(x) \propto \exp (x / 140 \mu \mathrm{m})$, for $x<0$, corresponds to Ref. [40]. We also show the maximal conversion efficiency for bremsstrahlung, achieved at optimal thickness for arbitrary target material, see Ref. [63]. The result for the dipole wave is shown with bounds corresponding to different phases. The conversion efficiency for the optimal phase is shown on the lower panel as a function of laser power $P$ and initial electron energy $\varepsilon_{0}$. The optimal laser power $P^{\text {opt }}$ is shown for each initial electron energy (dotted) together with the analytical estimate (dashed).

where $P^{\text {opt }}\left(\varepsilon_{0}\right)$ is the power that maximizes $N_{1 / 2} / N$ for a given electron energy $\varepsilon_{0}$.

The absolute values of conversion efficiency in this dipole field is obtained numerically and shown in Fig. 2 together with the results for several other shapes of the field. In these computations we assume that all particles propagate at the speed of light along a fixed direction $x$ and in a given field $S(x)$, influenced only by the processes of nonlinear Compton and multiphoton Breit-Wheeler. The QED processes are modeled with the adaptive event generator described in Ref. [20] with $\chi=\gamma S(x)$ (and similarly for the photons). Contributions from coherent higher order processes such as trident are neglected, as all field scale lengths are estimated to be much greater than the formation length in the considered regime $\left(\lambda / a_{0} \ll \lambda\right)$ [62,64-66]. The conversion efficiencies are presented in the top panel for optimal field amplitudes, determined individually for each field shape and initial electron energy by scanning for the optimal amplitude of $S(x)$. For the dipole field the variation due to the phase is presented as a band. In the lower panel we also show the conversion efficiency for the dipole wave as a function of both laser power $P$ and electron energy $\varepsilon_{0}$ together with the optimal laser power $P^{\text {opt }}$. One can see a reasonable agreement with the analytical estimates (1). It is notable that fairly accessible PW systems are capable of reaching efficiencies as high as that of bremsstrahlung, while in addition admitting high concentrations of generated photons and a clean environment for experiments.

Simulations.-To further assess the properties of the proposed source we turn to large-scale simulations, which were performed using the QED-PIC code ELMIS [20]. For the considered parameters the self-action of electrons is negligible. We, therefore, perform a set of simulations, each with fixed incoming laser power and given initial energy of electrons. By varying the incoming laser power and electron energy we obtain data that can be used to integrate the estimated yield for any given temporal variation of the field amplitude as well as any longitudinal variation of energy and density of electrons in the beam.

The simulations were carried out with a simulation box of $8 \mu \mathrm{m} \times 8 \mu \mathrm{m} \times 8 \mu \mathrm{m}$ divided into $128 \times 128 \times 128$ cells (this spatial resolution is sufficient since we do not consider regimes of dense plasma formation and its dynamics). The dipole field is generated at the boundary of this region with a wavelength $\lambda=1 \mu \mathrm{m}$ and cycle-averaged power $P$.

Electrons were injected into the simulation box along the dipole axis of symmetry and with given energy $\varepsilon_{0}$. This beam of electrons was modelled as having a Gaussian spatial envelope, with a FWHM waist $w=1 \mu \mathrm{m}$ and a FWHM length $L=5 \mu \mathrm{m}$, corresponding to a duration of $\tau_{0}=16.7 \mathrm{fs}$. The total charge of the beam was $100 \mathrm{pC}$, which translates into a total electron count of $N=$ $6.2 \times 10^{8}$, and a peak density of $10^{20} \mathrm{~cm}^{-3}$.

Apart from photons we gather statistics on all the particles, both those injected and generated through Breit-Wheeler pair creation. To extend the earlier definition of generation efficiency, we calculate the total number of photons $N_{x}$ above a given energy threshold $\left(\varepsilon_{\mathrm{th}}=x \gamma m c^{2}\right)$, for all photons escaping the interaction region.

Results-The photon generation efficiency is presented in Fig. 3 for different cutoff energies and as a function of $P$ and $\varepsilon_{0}$. It shows an intuitive trend for low cutoff energies [Fig. 3(a)], where both higher power and beam energy consistently translates into larger photon numbers, above the given threshold energy. However, for increasingly higher cutoffs [Fig. 3(b)] the efficiency instead displays an optimal laser power, for a given beam energy. This comes from the fact that as the laser power is increased, the pair production rate also increases. As a result, a smaller fraction of the high-energy photons escape the high-field region and instead fuel a shower-type cascade [67].

In Fig. 3(d) we show the efficiency for generating photons above half the initial electron beam energy. The high efficiency region at $\varepsilon_{0} \lesssim 1 \mathrm{GeV}$ is due to 

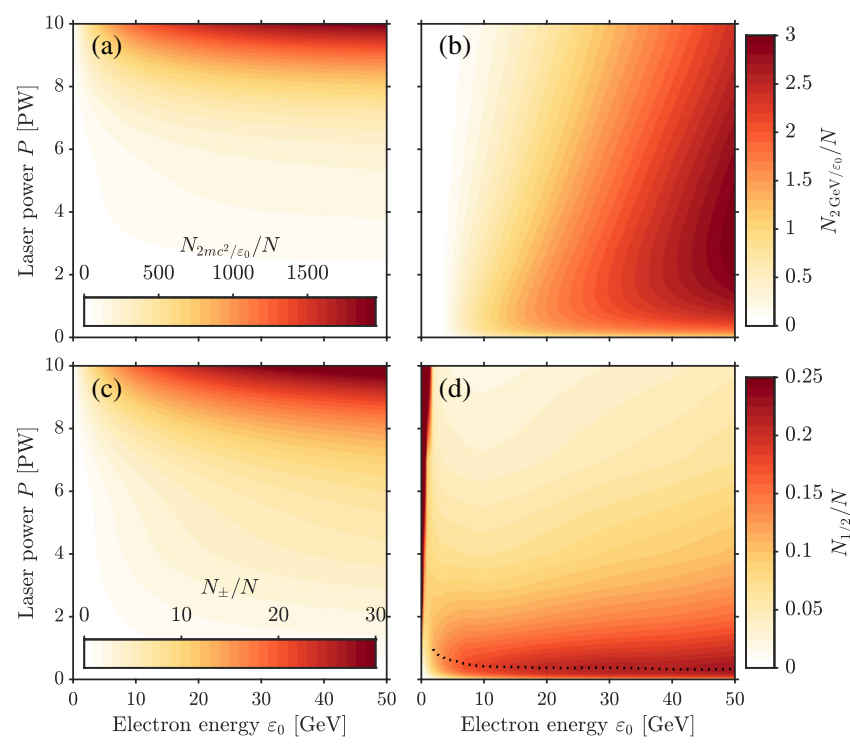

FIG. 3. Total number of photons detected above an energy threshold $\varepsilon_{\text {th }}$ of (a) $2 m c^{2}$, (b) $2 \mathrm{GeV}$, and (d) $\varepsilon_{0} / 2$, where $E$ is the electron beam energy. The values are normalized to the number of electrons in the beam $\left(N_{\varepsilon_{\mathrm{th}} / \varepsilon_{0}} / N\right)$. (c) Total number of generated electron-positron pairs at the end of the simulation, also normalized to the number of incoming electrons $\left(N_{ \pm} / N\right)$.

reacceleration in the laser field, which makes multiple emission of these photons possible [39]. Furthermore, there is also a region of high efficiency at large electron beam energies. As could already be seen from the high cutoff figure, in this region the efficiency initially increases with increasing laser power, but eventually drops off as the photon decay into pairs becomes dominant. The generation efficiency is here seen to be optimal around $0.4 \mathrm{PW}$, and with an electron beam energy of $10 \mathrm{GeV}$ it is possible to reach an efficiency of $18 \%$.

To further elucidate the interplay between the shower cascade and the suppression of high energy photons with increasing laser powers, it is informative to compare the photon and positron spectra for different laser powers and beam energies (Fig. 4). It can be seen that the number of photons above $1 \mathrm{GeV}$ is strongly suppressed for high laser powers, while the number of generated pairs increases, leading to the photon spectra for these laser powers to almost coincide for energies $>1 \mathrm{GeV}$.

The total number of pairs produced is similarly shown in Fig. 3(c). This shows a clear monotonic increase with both increasing $\varepsilon_{0}$ and $P$, as expected. For sufficiently large values we have a cascade. This region is also separated from both regions of high-energy photon production [compare Fig. 3(c) and 3(d)].

In Figs. 4(g)-4(j) we show energy-angle distribution of electrons and photons as they leave the interaction region. We note that some electrons still travel in the initial beam direction, and those electrons have the highest final energy. All other electrons, scattered by the EM field in all directions have much lower energies, limited by a several hundred $\mathrm{MeV}$ threshold. This can be explained by the fact that these electrons are moving in the radiation dominated regime. If we consider a strong rotating electric field, then the electron energy in such a field is given by $\left(a_{0} / \varepsilon_{\text {rad }}\right)^{1 / 4}$ [68], where $\varepsilon_{\mathrm{rad}}=4 \pi r_{e} / 3 \lambda$ and $r_{e}$ is the classical electron radius. This estimate works reasonably well for the case of a dipole wave, predicting maximum energies similar to that obtained in simulations.

Conclusions. - We have investigated the interaction of a highly energetic electron beam with an intense laser pulse,
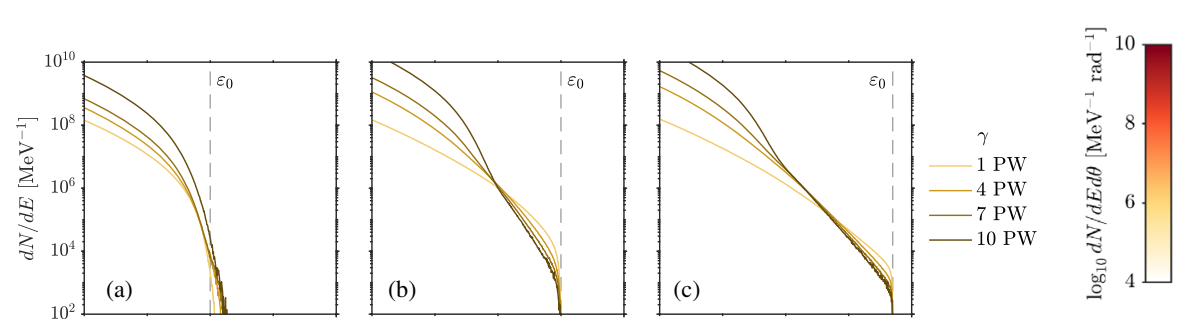

(g)
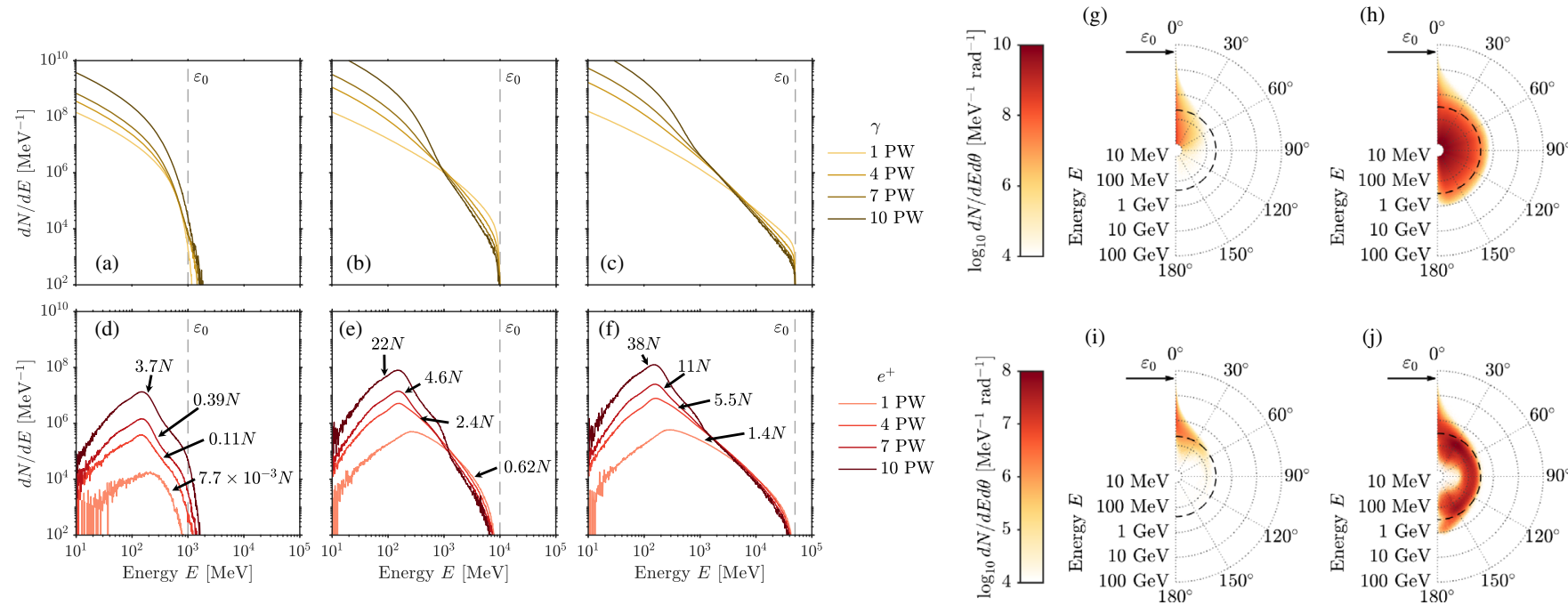

(i)

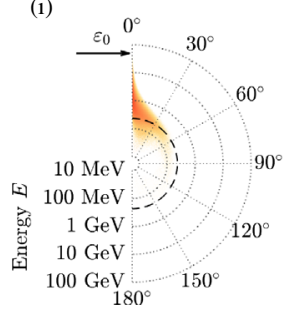

FIG. 4. Comparison between energy spectra of (a)-(c) photons (yellow) and (d)-(f) positrons (red) for different laser powers. Indicated with a dashed line is the initial electron energy: (a),(d) 1, (b),(e) 10, and (c),(f) $50 \mathrm{GeV}$. The total number of positrons is also indicated in (d)-(f), where $N$ is the total initial number of electrons in the beam. The energy-angle distribution of the generated (g)-(h) photons and (i)-(j) electrons are shown for an electron beam energy $\varepsilon_{0}$ of $50 \mathrm{GeV}$ and a laser power $P$ of (g), (i) 1 and (h), (j) 10 PW. 
in a geometry of optimal focusing, and assessed its capabilities as a source of $\mathrm{GeV}$-level photons. We find that increasing the laser power above $1 \mathrm{PW}$ leads to an increasingly stronger shower cascade, hampering the yield of high-energy photons. To efficiently generate photons above a few $\mathrm{GeV}$ we find that there is an optimal laser power of around $0.4 \mathrm{PW}$, around which it is possible to reach efficiencies in excess of $18 \%$. Assuming the capabilities of modern LWFA sources [69], this would lead to the production of multi-GeV photon beam, reaching a density of the order of $10^{18} \mathrm{~cm}^{-3}$. Note that at least partial polarization of photons can be achieved by letting the electron bunch pass the focused field off center, such that the radial acceleration of electrons leads to the presence of a predominant polarization direction.

Under such conditions, it would therefore be possible to use a significant amount of the power available to $10 \mathrm{PW}$ class systems to generate high-energy electron beams, having to dedicate only a smaller fraction to the photon generation. It also means that even with an imperfect geometry, it may still be possible to reach this regime with currently available laser powers, by compensating for the imperfections with a larger supplied power than suggested here.

J.M. would like to thank T. G. Blackburn for helpful discussions. S. S. B. acknowledges support from the Office of Science of the U.S. DOE under Contract No. DE-AC0205CH11231. J. K. K. acknowledges support from JSPS KAKENHI Grant No. 16K05639. S. V. B. acknowledges support at the ELI-BL by the project High Field Initiative (CZ.02.1.01/0.0/0.0/15 003/0000449) from European Regional Development Fund. The research is partly supported by the Ministry of Education and Science of the Russian Federation under Contract No. 14.W03.31.0032 (A. G., computations), by the Swedish Research Council Grants No. 2013-4248, 2016-03329 (M. M.) and 201705148 (A. G.), and by the Knut and Alice Wallenberg Foundation (A. G., J. M., M. M.). The simulations were performed on resources provided by the Swedish National Infrastructure for Computing (SNIC) at HPC2N.

*Corresponding author joel.magnusson@chalmers.se

[1] S. Gales et al., Rep. Prog. Phys. 81, 094301 (2018).

[2] ELI-Beamlines, https://www.eli-beams.eu.

[3] CORELS, https://corels.ibs.re.kr/.

[4] XCELS, http://www.xcels.iapras.ru.

[5] VULCAN, https://www.clf.stfc.ac.uk.

[6] C. Danson, D. Hillier, N. Hopps, and D. Neely, High Power Laser Sci. Eng. 3, e3 (2015).

[7] A. Di Piazza, K. Z. Hatsagortsyan, and C. H. Keitel, Phys. Rev. Lett. 105, 220403 (2010).

[8] S. V. Bulanov, T. Z. Esirkepov, Y. Hayashi, M. Kando, H. Kiriyama, J. K. Koga, K. Kondo, H. Kotaki, A. S. Pirozhkov, S. S. Bulanov, A. G. Zhidkov, P. Chen, D. Neely, Y. Kato,
N. B. Narozhny, and G. Korn, Nucl. Instrum. Methods Phys. Res., Sect. A 660, 31 (2011).

[9] A. Di Piazza, C. Müller, K. Z. Hatsagortsyan, and C. H. Keitel, Rev. Mod. Phys. 84, 1177 (2012).

[10] A. Ilderton and G. Torgrimsson, Phys. Rev. D 88, 025021 (2013).

[11] A. Ilderton and G. Torgrimsson, Phys. Lett. B 725, 481 (2013).

[12] M. Vranic, J. L. Martins, J. Vieira, R. A. Fonseca, and L. O. Silva, Phys. Rev. Lett. 113, 134801 (2014).

[13] D. Seipt, D. Del Sorbo, C. P. Ridgers, and A. G. R. Thomas, Phys. Rev. A 98, 023417 (2018).

[14] V. Y. Kharin, D. Seipt, and S. G. Rykovanov, Phys. Rev. Lett. 120, 044802 (2018).

[15] A. Gonoskov and M. Marklund, Phys. Plasmas 25, 093109 (2018).

[16] E. N. Nerush, I. Y. Kostyukov, A. M. Fedotov, N. B. Narozhny, N. V. Elkina, and H. Ruhl, Phys. Rev. Lett. 106, 035001 (2011).

[17] R. Duclous, J. G. Kirk, and A. R. Bell, Plasma Phys. Controlled Fusion 53, 015009 (2011).

[18] I. V. Sokolov, N. M. Naumova, and J. A. Nees, Phys. Plasmas 18, 093109 (2011).

[19] C. Ridgers, J. Kirk, R. Duclous, T. Blackburn, C. Brady, K. Bennett, T. Arber, and A. Bell, J. Comput. Phys. 260, 273 (2014).

[20] A. Gonoskov, S. Bastrakov, E. Efimenko, A. Ilderton, M. Marklund, I. Meyerov, A. Muraviev, A. Sergeev, I. Surmin, and E. Wallin, Phys. Rev. E 92, 023305 (2015).

[21] F. Niel, C. Riconda, F. Amiranoff, R. Duclous, and M. Grech, Phys. Rev. E 97, 043209 (2018).

[22] J. Derouillat, A. Beck, F. Pérez, T. Vinci, M. Chiaramello, A. Grassi, M. Flé, G. Bouchard, I. Plotnikov, N. Aunai, J. Dargent, C. Riconda, and M. Grech, Comput. Phys. Commun. 222, 351 (2018).

[23] N. Neitz and A. Di Piazza, Phys. Rev. Lett. 111, 054802 (2013).

[24] D. G. Green and C. N. Harvey, Phys. Rev. Lett. 112, 164801 (2014).

[25] T. Z. Esirkepov, S. S. Bulanov, J. K. Koga, M. Kando, K. Kondo, N. N. Rosanov, G. Korn, and S. V. Bulanov, Phys. Lett. A 379, 2044 (2015).

[26] C. S. Shen and D. White, Phys. Rev. Lett. 28, 455 (1972).

[27] C. N. Harvey, A. Gonoskov, A. Ilderton, and M. Marklund, Phys. Rev. Lett. 118, 105004 (2017).

[28] Y. B. Zel'dovich, Sov. Phys. Usp. 18, 79 (1975).

[29] L. L. Ji, A. Pukhov, I. Y. Kostyukov, B. F. Shen, and K. Akli, Phys. Rev. Lett. 112, 145003 (2014).

[30] J. G. Kirk, A. R. Bell, and I. Arka, Plasma Phys. Controlled Fusion 51, 085008 (2009).

[31] A. Gonoskov, A. Bashinov, I. Gonoskov, C. Harvey, A. Ilderton, A. Kim, M. Marklund, G. Mourou, and A. Sergeev, Phys. Rev. Lett. 113, 014801 (2014).

[32] M. Jirka, O. Klimo, S. V. Bulanov, T. Z. Esirkepov, E. Gelfer, S. S. Bulanov, S. Weber, and G. Korn, Phys. Rev. E 93, 023207 (2016).

[33] J. G. Kirk, Plasma Phys. Controlled Fusion 58, 085005 (2016).

[34] A. M. Fedotov, N. V. Elkina, E. G. Gelfer, N. B. Narozhny, and H. Ruhl, Phys. Rev. A 90, 053847 (2014).

[35] J. M. Cole et al., Phys. Rev. X 8, 011020 (2018). 
[36] K. Poder et al., Phys. Rev. X 8, 031004 (2018).

[37] M. Vranic, O. Klimo, G. Korn, and S. Weber, Sci. Rep. 8, 4702 (2018).

[38] M. Tamburini, A. Di Piazza, and C. H. Keitel, Sci. Rep. 7, 5694 (2017).

[39] A. Gonoskov, A. Bashinov, S. Bastrakov, E. Efimenko, A. Ilderton, A. Kim, M. Marklund, I. Meyerov, A. Muraviev, and A. Sergeev, Phys. Rev. X 7, 041003 (2017).

[40] A. Benedetti, M. Tamburini, and C. H. Keitel, Nat. Photonics 12, 319 (2018).

[41] D. J. Stark, T. Toncian, and A. V. Arefiev, Phys. Rev. Lett. 116, 185003 (2016).

[42] E. Wallin, A. Gonoskov, and M. Marklund, Phys. Plasmas 24, 093101 (2017).

[43] B. Lei, J. Wang, V. Kharin, M. Zepf, and S. Rykovanov, Phys. Rev. Lett. 120, 134801 (2018).

[44] O. Jansen, T. Wang, D. J. Stark, E. D’Humières, T. Toncian, and A. V. Arefiev, Plasma Phys. Controlled Fusion 60, 054006 (2018).

[45] E. S. Efimenko, A. V. Bashinov, S. I. Bastrakov, A. A. Gonoskov, A. A. Muraviev, I. B. Meyerov, A. V. Kim, and A. M. Sergeev, Sci. Rep. 8, 2329 (2018).

[46] E. S. Efimenko, A. V. Bashinov, A. A. Gonoskov, S. I. Bastrakov, A. A. Muraviev, I. B. Meyerov, A. V. Kim, and A. M. Sergeev, Phys. Rev. E 99, 031201 (2019).

[47] E. Esarey, B. A. Shadwick, P. Catravas, and W. P. Leemans, Phys. Rev. E 65, 056505 (2002).

[48] A. Rousse, K. T. Phuoc, R. Shah, A. Pukhov, E. Lefebvre, V. Malka, S. Kiselev, F. Burgy, J.-P. Rousseau, D. Umstadter, and D. Hulin, Phys. Rev. Lett. 93, 135005 (2004).

[49] H. Chen, S. C. Wilks, J. D. Bonlie, E. P. Liang, J. Myatt, D. F. Price, D. D. Meyerhofer, and P. Beiersdorfer, Phys. Rev. Lett. 102, 105001 (2009).

[50] F. Albert, A. G. R. Thomas, S. P. D. Mangles, S. Banerjee, S. Corde, A. Flacco, M. Litos, D. Neely, J. Vieira, Z. Najmudin, R. Bingham, C. Joshi, and T. Katsouleas, Plasma Phys. Controlled Fusion 56, 084015 (2014).

[51] W. P. Leemans, A. J. Gonsalves, H.-S. Mao, K. Nakamura, C. Benedetti, C. B. Schroeder, C. Tóth, J. Daniels, D. E. Mittelberger, S. S. Bulanov, J.-L. Vay, C. G. R. Geddes, and E. Esarey, Phys. Rev. Lett. 113, 245002 (2014).
[52] V. G. Nedorezov, A. A. Turinge, and Y. M. Shatunov, Phys. Usp. 47, 341 (2004).

[53] I. Gonoskov, A. Aiello, S. Heugel, and G. Leuchs, Phys. Rev. A 86, 053836 (2012).

[54] A. Golla, B. Chalopin, M. Bader, I. Harder, K. Mantel, R. Maiwald, N. Lindlein, M. Sondermann, and G. Leuchs, Eur. Phys. J. D 66, 190 (2012).

[55] L. Alber, M. Fischer, M. Bader, K. Mantel, M. Sondermann, and G. Leuchs, J. Eur. Opt. Soc. Publ. 13, 14 (2017).

[56] S. S. Bulanov, V. D. Mur, N. B. Narozhny, J. Nees, and V. S. Popov, Phys. Rev. Lett. 104, 220404 (2010).

[57] J. K. Koga and T. Hayakawa, Phys. Rev. Lett. 118, 204801 (2017).

[58] A. Gonoskov, I. Gonoskov, C. Harvey, A. Ilderton, A. Kim, M. Marklund, G. Mourou, and A. Sergeev, Phys. Rev. Lett. 111, 060404 (2013).

[59] E. G. Gelfer, A. A. Mironov, A. M. Fedotov, V. F. Bashmakov, E. N. Nerush, I. Y. Kostyukov, and N. B. Narozhny, Phys. Rev. A 92, 022113 (2015).

[60] M. Vranic, T. Grismayer, R. A. Fonseca, and L. O. Silva, Plasma Phys. Controlled Fusion 59, 014040 (2016).

[61] Z. Gong, R. H. Hu, Y. R. Shou, B. Qiao, C. E. Chen, X. T. He, S. S. Bulanov, T. Z. Esirkepov, S. V. Bulanov, and X. Q. Yan, Phys. Rev. E 95, 013210 (2017).

[62] A. I. Nikishov and V. I. Ritus, Sov. Phys. JETP 19, 529 (1964).

[63] T. G. Blackburn and M. Marklund, Plasma Phys. Controlled Fusion 60, 054009 (2018).

[64] B. King and H. Ruhl, Phys. Rev. D 88, 013005 (2013).

[65] V. Dinu and G. Torgrimsson, Phys. Rev. D 97, 036021 (2018).

[66] F. Mackenroth and A. Di Piazza, Phys. Rev. D 98, 116002 (2018).

[67] A. Mironov, N. Narozhny, and A. Fedotov, Phys. Lett. A 378, 3254 (2014).

[68] S. V. Bulanov, T. Z. Esirkepov, M. Kando, J. K. Koga, and S. S. Bulanov, Phys. Rev. E 84, 056605 (2011).

[69] A. J. Gonsalves et al., Phys. Rev. Lett. 122, 084801 (2019). 